\title{
GEOMETRY AND THE PETTIS INTEGRAL ${ }^{1}$
}

\author{
BY
}

ROBERT F. GEITZ

\begin{abstract}
Convex sets involving the range of a vector-valued function are constructed. These constructions provide a complete characterization of the bounded Pettis integrable functions.
\end{abstract}

In this paper we present a geometrical study of the Pettis integral. Our results show that there is a strong link, previously unsuspected, between the range of a vector-valued function and its properties of measurability and integrability. In the course of this paper we will characterize the functions that are equivalent to strongly measurable functions (Theorem 2.8), the bounded Pettis integrable functions on arbitrary finite measure spaces (Theorems 3.2 and 3.4), and the bounded Pettis integrable functions on perfect finite measure spaces (Theorem 4.6). Along the way we will develop new insights into the nature of weak measurability and the Pettis integral.

The link between measure theory and geometry in Banach spaces is not new. J. A. Clarkson defined the notion of uniform convexity almost fifty years ago as a condition to insure that functions of bounded variation are differentiable almost everywhere and have Bochner integrable derivatives. Radon-Nikodym theorems for the Bochner integral have been a fruitful source of geometrical properties. Rieffel introduced dentability in 1967 in the course of proving a Radon-Nikodym theorem. Slices and exposed points have similar origins. We are approaching measure theory from a different viewpoint from these authors. Rather than a global condition on the range space, we are looking for conditions on the range of an individual function that will guarantee that function to be integrable or measurable. This is in the spirit of the Pettis Measurability Theorem, which characterizes the weakly measurable functions that are strongly measurable in terms of the essential separability of their ranges.

1. Terminology. Let $(\Omega, \Sigma, \mu)$ be a finite measure space and let $X$ be a Banach space. A function $f(\cdot)$ from $\Omega$ into $X$ is weakly measurable if the scalar function $x^{*} f(\cdot)$ is measurable for each $x^{*}$ in the dual space $X^{*}$. The function $f$ is Pettis integrable if for each $E$ in $\Sigma$ there is an element of $X$, denoted $\int_{E} f d \mu$, that satisfies $x^{*} \int_{E} f d \mu=\int_{E} x^{*} f d \mu$ for every $x^{*}$ in $X^{*}$.

Received by the editors January 20, 1981. Parts of this paper were presented at the 786th meeting of the Society in Pittsburgh, Pennsylvania on May 16, 1981.

1980 Mathematics Subject Classification. Primary 28B05, 46G10.

Key words and phrases. Pettis integral, weak measurability, perfect measure, Banach space.

'This material constitutes a portion of the author's Ph.D. thesis from the University of Illinois under the direction of Professor J. J. Uhl, Jr. 
Another form of measurability can be attributed to some vector-valued functions. If $f$ is almost everywhere the limit (in the norm topology of $X$ ) of a sequence of simple functions, then $f$ is strongly measurable. The Pettis Measurability Theorem [9, Theorem 1.1] says that a function is strongly measurable if and only if it is weakly measurable and off a null set it has separable range. If $f$ is strongly measurable and if $\int\|f\| d \mu<\infty$ then the Bochner integral of $f$ exists as an element of $X$ [1, Theorem 2, p. 45], and $f$ is trivially Pettis integrable. The Bochner integral has received considerable study-see [1].

Functions $f$ and $g$ with values in $X$ are said to be equivalent if $x^{*} f=x^{*} g$ a.e. for every $x^{*}$ in $X^{*}$. Functions that are equivalent to strongly measurable functions need not themselves be strongly measurable. Example 2.3 gives a function $f$ for which $x^{*} f=0$ a.e. for each $x^{*}$ in $X^{*}$, yet $f$ is not strongly measurable.

2. The core. The principal tool in this section, the core, is related to Rieffel's notion of the essential range of a vector-valued function. After developing a few of the technical aspects of this tool, we use it to characterize the functions that are equivalent to strongly measurable functions.

Definition 2.1. Let $f: \Omega \rightarrow X$ and let $E \in \Sigma$. The core of $f$ over $E$, denoted $\operatorname{cor}_{f}(E)$, is the subset of $X$ given by the formula

$$
\operatorname{cor}_{f}(E)=\bigcap_{\mu(A)=0} \overline{\operatorname{co}} f(E \backslash A) .
$$

Our first theorem suggests that core is closely tied to the integrability of vector-valued functions.

TheOREM 2.2. If $f$ is a Pettis integrable function from $\Omega$ into $X$, then for each set $E$ in $\Sigma$

$$
\operatorname{cor}_{f}(E)=\operatorname{co}\left\{\frac{1}{\mu(B)} \int_{B} f d \mu: B \subset E, \mu(B)>0\right\}
$$

Proof. Both directions of this equality are valid precisely because they hold for scalar functions. First, let $B$ be a subset of $E$ of positive measure, and let $A$ be a null set. It follows easily from the Hahn-Banach theorem that

$$
\frac{1}{\mu(B)} \int_{B} f d \mu \in \overline{\operatorname{co}} f(B \backslash A) \subset \overline{\operatorname{co}} f(E \backslash A) \text {. }
$$

Hence $\overline{\operatorname{co}}\left\{(\mu(B))^{-1} \int_{B} f d \mu: B \subset E, \mu(B)>0\right\} \subset \operatorname{cor}_{f}(E)$.

For containment in the opposite direction, let $x$ be in $\operatorname{cor}_{f}(E)$, and let $\left\|x^{*}\right\|<1$. For any number $\varepsilon>0$ choose a countable partition $\pi$ of $E$ and a function $\alpha$, constant on the sets in $\pi$, such that the inequality $\left|x^{*} f(t)-\alpha(t)\right|<\varepsilon / 4$ holds for all $t$ in $E$. Note that if $B$ is any set in $\pi$ with positive measure and if $t \in B$ then, we must have the inequality

$$
\left|x^{*} f(t)-\frac{1}{\mu(B)} \int_{B} x^{*} f d \mu\right|<\varepsilon / 2 .
$$

Let $A$ be the union of the null sets in $\pi$. Then $x \in \overline{c o} f(E \backslash A)$; hence there is a finite convex sum $\sum \lambda_{i} f\left(t_{i}\right)$ such that $t_{i} \in E \backslash A$ and $\left\|x-\sum \lambda_{i} f\left(t_{i}\right)\right\|<\varepsilon / 2$. Thus 
we have

$$
\left|x^{*}(x)-\sum \lambda_{i} x^{*} f\left(t_{i}\right)\right|<\varepsilon / 2 .
$$

Finally, for each number $i$ let $B_{i}$ be the set in $\pi$ containing $t_{i}$. Observe that

$$
\left|x^{*}(x)-\sum \lambda_{i} \frac{1}{\mu\left(B_{i}\right)} \int_{B_{i}} x^{*} f d \mu\right|<\varepsilon .
$$

Since $\varepsilon$ is arbitrary, we see that

$$
x^{*}(x) \geqslant \inf \left[x^{*}\left\{\frac{1}{\mu(B)} \int_{B} f d \mu: B \subset E, \mu(B)>0\right\}\right] .
$$

It now follows from the Hahn-Banach theorem that

$$
x \in \overline{\operatorname{co}}\left\{(\mu(B))^{-1} \int_{B} f d \mu: B \subset E, \mu(B)>0\right\} .
$$

This completes the proof.

This theorem has several immediate consequences. For example, if $f$ is weakly equivalent to a strongly measurable function $g$, then $f$ has nonempty core. To see why, observe that inside any set of positive measure there is a subset $B$ such that $g \chi_{B}$ is bounded and Bochner integrable. It follows that $f \chi_{B}$ is Pettis integrable, and $\operatorname{cor}_{f}(B) \neq \varnothing$. Note that if $f$ and $g$ are weakly equivalent Pettis integrable functions, then they have the same core, since this implies $\int_{B} f d \mu=\int_{B} g d \mu$ for each measurable set $B$. A later theorem (Theorem 2.6) will show that this does not depend on the integrability of $f$ and $g$; equivalent functions always have the same core.

Before exploring more fully the properties of the core, we present two examples. The first of these is an easy example that illustrates the utility of the core.

EXAMPLE 2.3. A function with nonseparable range whose core consists of a single point. Let $l_{2}[0,1]$ be the usual space of countably nonzero functions on $[0,1]$ that are square-summable, under the $l_{2}$-norm. For each point $t$ in $[0,1]$ define $e_{t} \in$ $l_{2}[0,1]$ by

$$
e_{t}(s)= \begin{cases}1 & \text { if } s=t \\ 0 & \text { if } s \neq t\end{cases}
$$

Define $f$ from $[0,1]$ into $l_{2}[0,1]$ by $f(t)=e_{t}$ for each $t$ in $[0,1]$. This function has nonseparable range; in fact, the span of its range is dense in the nonseparable space $l_{2}[0,1]$. However, since $\left(l_{2}[0,1]\right)^{*}=l_{2}[0,1]$, the function $x^{*} f$ is only countably nonzero for each $x^{*}$ in $X^{*}$. Hence $x^{*} f=0$ a.e. (with respect to Lebesgue measure,) and $f$ is weakly equivalent to the zero function. The core of $f$ over any set of positive measure thus contains only the zero element of $l_{2}[0,1]$.

The second example is much more complicated. This is Phillips's example [10, Example 10.8] of a bounded weakly measurable function that is not Pettis integrable.

EXAMPLE 2.4. $A$ bounded nonintegrable function with empty core. Let $l_{\infty}[0,1]$ be the usual space of all bounded real-valued functions on $[0,1]$, equipped with the 
supremum norm. Under the assumption of the continuum hypothesis, Sierpinski constructed a subset $B$ of the unit square $[0,1] \times[0,1]$ with properties

(1) for each number $t_{0}$ in $[0,1]$, the set $\left\{s:\left(s, t_{0}\right) \in B\right\}$ is countable and

(2) for each number $s_{0}$ in $[0,1]$, the set $\left\{t:\left(s_{0}, t\right) \notin B\right\}$ is countable.

Let $(\Omega, \Sigma, \mu)$ be the standard Lebesgue measure space on $[0,1]$, and define a function $f$ from $[0,1]$ into $l_{\infty}[0,1]$ by

$$
[f(s)](t)=\chi_{B}(s, t) .
$$

Phillips has shown that $f$ is weakly measurable. In fact, for each $x^{*}$ in $l_{\infty}^{*}[0,1], x^{*} f$ is almost everywhere constant.

We now show that the core of $f$ is empty; hence $f$ is not Pettis integrable. For each number $t$ let $A_{t}$ be the set $A_{t}=\{s:(s, t) \in B\}$. The properties of $B$ imply that $\mu\left(A_{t}\right)=0$ for each $t$. Observe that if $s \notin A_{t}$ then $[f(s)](t)=0$. Hence, if $x \in$ $\overline{\operatorname{co}} f\left(\Omega \backslash A_{t}\right)$ then $x(t)=0$. If $x \in \operatorname{cor}_{f}(\Omega)$, then we must have $x(t)=0$ for every $t$; the only possible element of $\operatorname{cor}_{f}(\Omega)$ is the zero element of $l_{\infty}[0,1]$. However, if $\sum \lambda_{i} f\left(s_{i}\right)$ is a finite convex sum, in which $s_{i} \in \Omega$ for every $i$, then

$$
\left\|\sum \lambda_{i} f\left(s_{i}\right)\right\|=\sup _{t}\left|\sum \lambda_{i} \chi_{B}\left(s_{i}, t\right)\right|=1 \text {. }
$$

Hence $0 \notin \overline{\operatorname{co}} f(\Omega)$, and $f$ has an empty core.

We now pass to the study of equivalence to strong measurability. We require the following elementary lemma, whose proof we omit.

LEMMA 2.5. Let $\alpha$ and $\beta$ be measurable scalar-valued functions, and let $\mu(E)>0$. If $\alpha(t)<\beta(t)$ for all $t$ in $E$ then there is a set $E^{*} \subset E$ such that $\mu\left(E^{*}\right)>0$ and

$$
\sup \alpha\left(E^{*}\right)<\inf \beta\left(E^{*}\right) \text {. }
$$

We come now to an important result; Theorem 2.6 has many applications.

THEOREM 2.6. Let $f$ and $g$ be weakly measurable functions from $\Omega$ into $X$. If $x^{*} f=x^{*} g$ a.e. for all $x^{*}$ in $X^{*}$, then $\operatorname{cor}_{f}(E)=\operatorname{cor}_{g}(E)$ for every measurable set $E$. Conversely, if both $f$ and $g$ have nonempty cores and if $\operatorname{cor}_{f}(E)=\operatorname{cor}_{g}(E)$ for every $E$ in $\Sigma$ then $x^{*} f=x^{*} g$ a.e. for all $x^{*}$ in $X^{*}$.

Proof. First suppose that $x^{*} f=x^{*} g$. a.e. for all $x^{*}$ in $X^{*}$. Let $x$ be any element of $\operatorname{cor}_{f}(E)$, and let $A_{0}$ be any null set. It suffices to show that for each $x^{*}$ in $X^{*}$, $x^{*}(x) \geqslant \inf x^{*} g\left(E \backslash A_{0}\right)$. To this end, fix $x^{*}$ and let $A_{1}=A_{0} \cup\left\{t: x^{*} f(t) \neq\right.$ $\left.x^{*} g(t)\right\}$. Clearly $\mu\left(A_{1}\right)=0$; hence $x \in \overline{\operatorname{co}} f\left(E \backslash A_{1}\right)$, and

$$
\begin{aligned}
x^{*}(x) & \geqslant \inf x^{*} f\left(E \backslash A_{1}\right) \\
& =\inf x^{*} g\left(E \backslash A_{1}\right) \geqslant \inf x^{*} g\left(E \backslash A_{0}\right) .
\end{aligned}
$$

For the second statement, suppose there is a functional $x^{*}$ in $X^{*}$ such that the condition of $x^{*} f=x^{*} g$ a.e. fails. We may assume without loss of generality that

$$
\mu\left[t: x^{*} f(t)<x^{*} g(t)\right]>0 .
$$

By the lemma, there must be a set $E^{*}$ of positive measure such that $\sup x^{*} f\left(E^{*}\right)<$ inf $x^{*} g\left(E^{*}\right)$. The sets $\overline{\operatorname{co}} f\left(E^{*}\right)$ and $\overline{\operatorname{co}} g\left(E^{*}\right)$ are thus disjoint, and the equality $\operatorname{cor}_{f}(E)=\operatorname{cor}_{g}(E)$ can only hold vacuously, that is, if the cores of both $f$ and $g$ are void. 
Theorem 2.6 points out the utility of the notion of the core: this notion equates functions that the linear functionals believe are essentially the same. A function can have a very large range and still be weakly equivalent to a very simple function, as Example 2.3 shows. Thanks to this theorem, we can study equivalence to strong measurability by studying the ranges of strongly measurable functions. As a first step in this direction, we state a lemma that is well known.

LEMMA 2.7. A function $g: \Omega \rightarrow X$ is strongly measurable if and only if for each set $E$ of positive measure and for each $\varepsilon>0$ there is a measurable set $E^{\prime} \subset E$ such that $\mu\left(E^{\prime}\right)>0$ and $\operatorname{diam}\left[g\left(E^{\prime}\right)\right]<\varepsilon$.

An immediate consequence of Theorem 2.6 and this lemma is that if $f$ is equivalent to a strongly measurable function then inside every set there is a subset over which the core of $f$ has small diameter. We now show that the converse of this statement is also true. The next theorem completely characterizes equivalence to strong measurability.

THEOREM 2.8. A weakly measurable function $f: \Omega \rightarrow X$ is equivalent to a strongly measurable function if and only if for each set $E$ of positive measure and for each $\varepsilon>0$ there is a set $E^{\prime} \subset E$ such that $\mu(E)>0, \operatorname{cor}_{f}(E) \neq \varnothing$, and $\operatorname{diam}\left[\operatorname{cor}_{f}(E)\right]$ $<\varepsilon$.

Proof. One direction of this is an immediate consequence of the preceding theorem and lemma. For the converse, we assume that $f$ has the stated property and construct a strongly measurable function $g$ that is equivalent to $f$. For each integer $n$ the method of exhaustion [1, Lemma III.2.4] produces a countable partition $\pi_{n}$ of $\Omega$ such that, for every set $B \in \pi_{n}$, diam[ $\left.\operatorname{cor}_{f}(B)\right]<1 / n$. We assume that $\pi_{n}$ refines $\pi_{m}$ if $n>m$. For each $n$ and for each $B$ in $\pi_{n}$, let $x_{B} \in \operatorname{cor}_{f}(B)$. Define a sequence $\left(f_{n}\right)$ of countably valued functions by $f_{n}(t)=\sum_{B \in \pi_{n}} x_{B} \chi_{B}(t)$. These functions have been selected in such a way that for almost all $t$ in $\Omega$ the sequence $\left\{f_{n}(t)\right\}$ is norm Cauchy. The formula $g(t)=\lim _{n} f_{n}(t)$ defines a strongly measurable function $g: \Omega \rightarrow X$.

It only remains to show that $f$ is weakly equivalent to $g$. If these functions are not equivalent, then there is a functional $x^{*}$ in $X^{*}$ and a set $B$ such that $\mu(B)>0$ and $x^{*} f(t)>x^{*} g(t)$ for all $t$ in $B$. With the help of Lemma 2.5 we can find a subset $B^{\prime} \subset B$ of positive measure and a number $\varepsilon>0$ such that

$$
\inf \left[x^{*} f\left(B^{\prime}\right)\right]>\sup \left[x^{*} g\left(B^{\prime}\right)\right]+\varepsilon .
$$

We may assume that the sequence $\left(f_{n}\right)$ converges to $g$ uniformly on $B^{\prime}$. Finally, pick $n$ so large that $n>2 / \varepsilon$ and $\left\|f_{n}(t)-g(t)\right\|<\varepsilon / 2$ for all $t$ in $B^{\prime}$. Pick a set $D$ in $\pi_{n}$ such that $\mu\left(B^{\prime} \cap D\right)>0$; let $t \in B^{\prime} A D$, and let $x$ be any point in the set $\operatorname{cor}_{f}\left(B^{\prime} \cap D\right)$. Then $x$ and $f_{n}(t)$ are both in the set $\operatorname{cor}_{f}(D)$, which has diameter less than $\varepsilon / 2$; hence $\left\|x-f_{n}(t)\right\|<\varepsilon / 2$. This proves that $\|x-g(t)\|<\varepsilon$, and that $x^{*}(x)<x^{*} g(t)+\varepsilon$. However, we have

$$
x^{*} g(t) \leqslant \sup \left[x^{*}\left(g\left(B^{\prime}\right)\right)\right]
$$

and since $x \in \overline{\operatorname{co}} f\left(B^{\prime}\right)$, it follows that $x^{*}(x) \geqslant \inf \left[x^{*} f\left(B^{\prime}\right)\right]$. This shows that

$$
\inf \left[x^{*} f\left(B^{\prime}\right)\right] \leqslant \sup \left[x^{*} g\left(B^{\prime}\right)\right]+\varepsilon,
$$


in contradiction to inequality (*), and thus for each $x^{*}$ in $X^{*}$

$$
\mu\left[t: x^{*} f(t) \neq x^{*} g(t)\right]=0 .
$$

Lemma 2.7 and Theorem 2.8 may be summarized in the following terminology. A weakly measurable function is strongly measurable if and only if it has locally small range, and is equivalent to a strongly measurable function if and only if has locally small core. As an illustration of this, for each Lebesgue measurable set $E$ of positive measure the function $f$ in Example 2.3 has $\operatorname{diam}[f(E)]=\sqrt{2}$, but $\operatorname{cor}_{f}(E)$ consists only of a single point. In a sense, the intersections that form the core pare the range of a function down to its bare essentials. A function can have a very complicated range but still be simple from a measure-theoretic point of view. The core allows us to see the range of such a function in its simplest state.

Before leaving the core, we note the following connection between it and a well-known set function. Suppose $f: \Omega \rightarrow X$ is strongly measurable and $E$ is in $\Sigma$. Rieffel has defined the essential range of $f$ over $E, r_{f}(E)$, as the collection of all elements $x$ in $X$ such that for each $\varepsilon>0$ the set $\{t \in E:\|f(t)-x\|<\varepsilon\}$ has positive measure. Our concept of core is closely related to this notion. A straightforward calculation places essential range in a geometrical setting: If $f$ is strongly measurable then

$$
r_{f}(E)=\bigcap_{\mu(A)=0} \overline{f(E \backslash A)} .
$$

After noting the similarity between this and the definition of $\operatorname{cor}_{f}(E)$, the following theorem is not surprising.

THEOREM 2.9. If $f: \Omega \rightarrow X$ is strongly measurable, then for every set $E$ in $\Sigma$

$$
\operatorname{cor}_{f}(E)=\overline{\operatorname{cor}}\left[r_{f}(E)\right] \text {. }
$$

The proof is not difficult and is omitted.

3. Pettis integrability on finite measure spaces. In this section we present two characterizations of the Pettis integrable functions. These theorems give the only known necessary and sufficient conditions for Pettis integrability with respect to an arbitrary finite measure.

Definition 3.1. Let $f: \Omega \rightarrow X$, let $B \subset \Omega$ be a measurable set, and let $\pi$ be a finite partition of $B$ into measurable sets. We denote by $S(\pi, B)$ the closure of the convex set $\Sigma_{E \in \pi} \overline{\operatorname{co}} f(E) \mu(E)$.

Now suppose that $f: \Omega \rightarrow X$ is Pettis integrable. If $B \in \Sigma$ and if $\pi$ is a finite partition of $B$, then

$$
\int_{B} f d \mu=\sum_{E \in \pi} \int_{e} f d \mu \in \sum_{E \in \pi} \overline{\operatorname{co}} f(E) \mu(E) .
$$

In particular $\int_{B} f d \mu \in S(\pi, B)$, and in fact $\int_{B} f d \mu \in \cap_{\pi} S(\pi, B)$, where the intersection is taken over all partitions $\pi$ of $B$. Our first theorem shows that these sets $S(\pi, B)$ play a central role in the study of the Pettis integrability of an individual function. 
TheOREM 3.2. A bounded weakly measurable function $f: \Omega \rightarrow X$ is Pettis integrable if and only if $\cap_{\pi} S(\pi, B) \neq \varnothing$ for each measurable set $B$.

Proof. The remarks preceding the statement of the theorem show that $\int_{B} f d \mu \in$ $\cap_{\pi} S(\pi, B)$ if $f$ is Pettis integrable.

For the converse, we will show that $\int_{B} f d \mu$ is the only possible element of $\cap_{\pi} S(\pi, B)$. Fix $B$ in $\Sigma$ and assume that $x \in \cap_{\pi} S(\pi, B)$; we shall show that $x^{*}(x)=\int_{B} x^{*} f d \mu$ for every $x^{*}$ in $X^{*}$. Fix such an $x^{*}$ and let $\varepsilon>0$. Since the scalar-valued function $x^{*} f$ is bounded, it is the uniform limit of a sequence of simple functions. It follows that there is a finite partition $\pi$ of $B$ such that if $E$ is in $\pi$ and if $t_{1}$ and $t_{2}$ are points in $E$, then

$$
\left|x^{*} f\left(t_{1}\right)-x^{*} f\left(t_{2}\right)\right|<\varepsilon .
$$

Hence for any point $t$ in $E$, we have

$$
\left|\frac{1}{\mu(E)} \int_{E} x^{*} f d \mu-x^{*} f(t)\right|<\varepsilon,
$$

and

$$
\left|\int_{E} x^{*} f d \mu-x^{*} f(t) \mu(E)\right|<\varepsilon \mu(E) .
$$

If $\sum \alpha_{i} f\left(t_{i}\right)$ is a convex sum with each $t_{i}$ in $E$, then

$$
\left|\int_{E} x^{*} f d \mu-x^{*}\left(\sum \alpha_{i} f\left(t_{i}\right)\right) \mu(E)\right|<\varepsilon \mu(E) .
$$

It follows that if $x_{E} \in \overline{\operatorname{co}} f(E)$ for each $E$ in $\pi$, then

$$
\left|\int_{E} x^{*} f d \mu-x^{*}\left(x_{E}\right) \mu(E)\right| \leqslant \varepsilon \mu(E) .
$$

Summing over all the sets $E$ in $\pi$ gives $\left|\int_{B} x^{*} f d \mu-x^{*}(x)\right| \leqslant \varepsilon \mu(B)$. Since this holds for each $\varepsilon>0$, we have $x^{*}(x)=\int_{B} x^{*} f d \mu$, and

$$
x=\text { Pettis- } \int_{B} f d \mu .
$$

One way to insure that the sets $S(\pi, B)$ have nonvoid intersection is to require them to meet a weakly compact set.

COROLlary 3.3. A bounded weakly measurable function $f: \Omega \rightarrow X$ is Pettis integrable if and only if for each set $B$ in $\Sigma$ there is a weakly compact set $W \subset X$ such that $W \cap S(\pi, B) \neq \varnothing$ for every finite partition $\pi$ of $B$.

Proof. If $f$ is Pettis integrable, let $W$ be the weak closure of the set $\left\{\int_{E} f d \mu\right.$ : $E \in \Sigma$ \}. This set is well known to be weakly compact (see [1, Corollary II.3.9]) and we have already seen that $W \cap S(\pi, B) \neq \varnothing$ for every $\pi$ and $B$.

To prove the opposite implication, observe that if $\pi$ and $\pi^{\prime}$ are partitions of $B$ such that $\pi^{\prime}$ refines $\pi$, then $S\left(\pi^{\prime}, B\right) \subset S(\pi, B)$. Hence the family of sets $\{W \cap$ $S(\pi, B): \pi$ is a portion of $B\}$ has the finite intersection property. Since $W$ is weakly compact and these sets are weakly closed, this family has nonempty intersection. This holds for each $B$ in $\Sigma$; by Theorem 3.2 is Pettis integrable. 
Our second characterization looks back to Theorem 2.2, where we showed that the average values of a Pettis integrable function lie in its core. We now show that the indefinite Pettis integral is the only vector measure $F$ such that $F(E)$ $\in \overline{\operatorname{co}} f(E) \mu(D)$ for every measurable set $E$.

THEOREM 3.4. A bounded weakly measurable function $f: \Omega \rightarrow X$ is Pettis integrable if and only if there is a finitely additive vector measure $F: \Sigma \rightarrow X$ such that

$$
F(E) \in \overline{\operatorname{co}} f(E) \mu(E)
$$

for every set $E$ in $\Sigma$.

Proof. Suppose $f$ is Pettis integrable and write $F(E)=\int_{E} f d \mu$ for $E$ in $\Sigma$. Appeal to [1, Theorem II.3.5] to see that $F$ is a countable additive vector measure. A glance at the introduction to this section shows that $F(E) \in \overline{\operatorname{co}} f(E) \mu(E)$.

To prove the converse, note that for any set $B$ in $\Sigma$ and any finite partition $\pi$ of $B$,

$$
F(B)=\sum_{E \in \pi} F(E) \in \sum_{E \in \pi} \overline{\operatorname{co}} f(E) \mu(E) \subset S(\pi, B) .
$$

The conclusion now follows from Theorem 3.2.

4. Integration on perfect measure spaces. In this section we restrict our attention to integration on perfect measure spaces. This is a very broad class of measure spaces first defined by Gnedenko and Kolmogorov for the purpose of avoiding pathological behavior in probability theory. They were the subject of an extensive study by Sazonov [12] and form the background for a major work by Fremlin [6]. As we show in [7] and in the present work, they also eliminate much of the pathological behavior of the Pettis integral.

Charles Stegall has shown [6, Proposition 3J] that the indefinite Pettis integral with respect to a perfect measure has relatively norm compact range. This strengthens one direction of Corollary 3.3. If $(\Omega, \Sigma, \mu)$ is perfect and if $f: \Omega \rightarrow X$ is bounded and Pettis integrable, then let $K$ be the norm closure of the set $\left\{\int_{E} F d \mu\right.$ : $E \in \Sigma\}$. Stegall's theorem shows that $K$ is compact, and clearly $K \cap S(\pi, B) \neq \varnothing$ for every partition $\pi$ of $B$. We thus have the following.

THEOREM 4.1. Let $(\Omega, \Sigma, \mu)$ be a perfect measure space, and let $f: \Omega \rightarrow X$ be $a$ weakly measurable function. Each of the following statements implies all the others.

(1) $f$ is Pettis integrable.

(2) For each $b$ in $\Sigma$ there is a weakly compact set $W \subset X$ such that $W \cap S(\pi, B)$ $\neq \varnothing$ for every partition $\pi$ of $B$.

(3) For each $B$ in $\Sigma$ there is a norm compact set $K \subset X$ such that $K \cap S(\pi, B) \neq$ $\varnothing$ for every partition $\pi$ of $B$.

In the remainder of this section we consider three set functions whose values are contained in the core of $f$. Since we are considering only bounded functions, without loss of generality we may assume $\|f(t)\| \leqslant 1$ for all $t$. The definitions below may be easily altered to encompass arbitrary bounded functions. 
Definition 4.2. Let $f$ be a function from $\Omega$ into the unit ball $U$ of $X$ and let $E$ be a measurable set. The sets $G_{f}(E)$ and $H_{f}(E)$ are defined by

$$
G_{f}(E)=\bigcap_{A \subset E} \overline{\operatorname{co}}[f(E \backslash A) \mu(E \backslash A)+\mu(A) U],
$$

and

$$
H_{f}(E)=\bigcap_{A \subset E} \overline{\operatorname{co}}[f(E \backslash A) \mu(E)+3 \mu(A) U],
$$

where the intersections are taken over all measurable proper subsets of $E$. The set $Q_{f}(E)$ consists of those $x$ in $X$ such that the inequality

$$
\mu\left[t \in E: x^{*} f(t) \leqslant x^{*}(X)+\varepsilon\right]>\varepsilon \mu(E) / 2
$$

holds whenever $\left\|x^{*}\right\| \leqslant 1$ and $0<\varepsilon<2$.

It is immediately clear that these sets are convex, that $G_{f}(E)$ and $H_{f}(E)$ are closed, that $G_{f}(E) \subset H_{f}(E) \subset \operatorname{cor}_{f}(E)$, and that $\int_{E} f d \mu \in G_{f}(E)$ whenever $f$ is Pettis integrable. We shall see later that $G_{f}(E) \subset Q_{f}(E) \mu(E) \subset H_{f}(E)$, but that $Q_{f}(E)$ is not necessarily closed. These sets are therefore distinct. We are now going to show that when $(\Omega, \Sigma, \mu)$ is perfect these sets have two surprising properties.

(1) for every $E$ in $\Sigma$ the sets $G_{f}(E)$ and $H_{f}(E)$ are compact, and

(2) the function $f$ is Pettis integrable whenever $G_{f}(E)$ and $H_{f}(E)$ are nonempty for all sets $E$ of positive measure.

The first lemma for this is an analogue for the norm topology of a classic theorem of R. C. James.

LEMMA 4.3. Let $C$ be a closed bounded set in $X$. If $C$ is not norm compact then there is a number $\varepsilon>0$ and there are sequences $\left(x_{k}\right)$ in $C$ and $\left(x_{n}^{*}\right)$ in the unit ball of $X^{*}$ such that $x_{n}^{*}\left(x_{n}\right) \geqslant \varepsilon$ and $x_{n}^{*}\left(x_{k}\right)=0$ when $k<n$.

Proof. Select a number $\varepsilon>0$ such that $C$ cannot be covered by finitely many balls of radius $2 \varepsilon$. Inductively select a sequence $\left(x_{k}\right)$ in $C$ such that

$$
\operatorname{dist}\left(x_{n+1}, \operatorname{span}\left[x_{1}, \ldots, x_{n}\right]\right)>\varepsilon
$$

for all integers $n$ as follows. Let $x_{1}$ be any element of $C$. If $x_{1}, \ldots, x_{n-1}$ are elements of $C$ that have been chosen to satisfy (*), then there must be an element of $C$ not in the set $\operatorname{span}\left[x_{1}, \ldots, x_{n-1}\right]+\varepsilon U$, or else $C$ could be covered by finitely many $2 \varepsilon$ balls. Let this point be $x_{n}$, and let $d_{n}$ be the distance from $x_{n}$ to $\operatorname{span}\left[x_{1}, \ldots, x_{n-1}\right]$; clearly $d_{n}>\varepsilon$. Observe that the sequence $\left(x_{k}\right)$ thus selected is linearly independent. For each integer $n$ we define a functional $\psi_{n}$ on $\operatorname{span}\left[x_{1}, \ldots, x_{n}\right]$ by

$$
\psi_{n}\left(\sum_{i=1}^{n} \alpha_{i} x_{i}\right)=d_{n} \alpha_{n}
$$

for all real numbers $\alpha_{1}, \ldots, \alpha_{n}$. An easy calculation shows that $\left\|\psi_{n}\right\| \leqslant 1$. The Hahn-Banach theorem extends $\psi_{n}$ to an element $x_{n}^{*}$ of the unit ball of $X^{*}$, and this completes the proof. 
TheOREM 4.4. Let $(\Omega, \Sigma, \mu)$ be a finite perfect measure space and let $E \in \Sigma$. If $f$ is a bounded weakly measurable function from $\Omega$ into the unit ball of $X$ then $H_{f}(E)$ is compact.

Proof. If $H_{f}(E)$ is not compact then by Lemma 4.3 we may select a sequence $\left(x_{k}\right)$ in $H_{f}(E)$, a bounded sequence $\left(x_{n}^{*}\right)$ in $X^{*}$, and a number $\varepsilon>0$ such that $x_{n}^{*}\left(x_{n}\right)>\varepsilon$ for all $n$, and $x_{n}^{*}\left(x_{k}\right)=0$ for $k<n$. Applying [7, Lemma 1] to the sequence $\left(x_{n}^{*} f\right)$, we may extract a subsequence $\left(x_{n_{n}}^{*} f\right)$ and find a weak ${ }^{*}$ cluster point $x_{0}^{*}$ of $\left(x_{n_{j}}^{*}\right)$ such that $\lim _{j} x_{n_{j}}^{*} f=x_{0}^{*} f$ a.e. Egorov's theorem produces a set $A$ such that $\left(x_{n_{j}}^{*} f\right)$ converges uniformly on $E \backslash A$ and $\mu(A)<\varepsilon / 10$. Let $m_{1}$ and $m_{2}>m_{1}$ be any two indices for this subsequence such that the inequality

$$
\left|x_{0}^{*} f(t)-x_{m_{i}}^{*} f(t)\right|<\varepsilon / 10 \mu(E)
$$

holds for all $t$ in $E \backslash A$ and for $i=1,2$. Since

$$
x_{m_{1}} \in \overline{\mathrm{co}}[f(E \backslash A) \mu(E)+3 \mu(A) U],
$$

there is a convex sum $\sum_{i=1}^{n} \alpha_{i} f\left(t_{i}\right)$ such that each $t_{i}$ is in $E \backslash A$ and

$$
\left\|x_{m_{1}}-\mu(E) \sum_{i=1}^{n} \alpha_{i} f\left(t_{i}\right)\right\| \leqslant \varepsilon / 10+3 \mu(A) \leqslant 2 \varepsilon / 5 .
$$

Hence, for all $x^{*}$ in the unit ball of $X^{*}$,

$$
\left|x^{*}\left(x_{n_{1}}\right)-\mu(E) \sum_{i=1}^{n} \alpha_{i} x^{*} f\left(t_{i}\right)\right| \leqslant 2 \varepsilon / 5 .
$$

To produce a contradiction, we first let $x^{*}=x_{m_{1}}^{*}$. Since $\left|x_{m_{1}}^{*} f\left(t_{i}\right)-x_{0}^{*} f\left(t_{i}\right)\right|<$ $\varepsilon / 10 \mu(E)$ holds for $1 \leqslant i \leqslant n$, the inequality

$$
\left|x_{m_{1}}^{*}\left(x_{m_{1}}\right)-\mu(E) \sum_{i=1}^{n} \alpha_{i} x_{0}^{*} f\left(t_{i}\right)\right| \leqslant \varepsilon / 10+2 \varepsilon / 5=\varepsilon / 2
$$

holds. Since $x_{m_{1}}^{*}\left(x_{m_{1}}\right)>\varepsilon$, we see that $\left|\mu(E) \sum_{i=1}^{n} \alpha_{i} x_{0}^{*} f\left(t_{i}\right)\right| \leqslant \varepsilon / 2$. On the other hand, letting $x^{*}=x_{m_{2}}^{*}$ in (*) yields $\left|\mu(E) \sum_{i=1}^{n} \alpha_{i} x_{m_{2}}^{*}\right| \leqslant 2 \varepsilon / 5$; hence

$$
\left|\mu(E) \sum_{i=1}^{n} \alpha_{i} x_{0}^{*} f\left(t_{i}\right)\right| \leqslant \varepsilon / 2 .
$$

This is a contradiction and proves that $H_{f}(E)$ must be compact.

LEMMA 4.5. $H_{f}$ is superadditive, i.e.,

$$
H_{f}\left(E_{1}\right)+H_{f}\left(E_{2}\right) \subset H_{f}\left(E_{1} \cup E_{2}\right)
$$

whenever $E_{1}$ and $E_{2}$ are disjoint measurable sets.

Proof. Let $x_{i} \in H_{f}\left(E_{i}\right)$ for $i=1,2$, and let $A \subset E_{1} \cup E_{2}$. We must show that

$$
x_{1}+x_{2} \in \overline{\mathrm{co}}\left[f\left(E_{1} \cup E_{2} \backslash A\right) \mu\left(E_{1} \cup E_{2}\right)+3 \mu(A) U\right] \text {. }
$$

Case 1. If $E_{1} \backslash A \neq \varnothing$ and $E_{2} \backslash A \neq \varnothing$ then

$$
\begin{aligned}
x_{1}+x_{2} \in & \overline{\operatorname{co}}\left[f\left(E_{1} \backslash A\right) \mu\left(E_{1}\right)+3 \mu\left(A \cap E_{1}\right) U\right] \\
& +\overline{\operatorname{co}}\left[f\left(E_{2} \backslash A\right) \mu\left(E_{2}\right)+3 \mu\left(A \cap E_{2}\right) U\right] \\
\subset & \operatorname{co}\left[f\left(E_{1} \cup E_{2} \backslash A\right) \mu\left(E_{1} \cup E_{2}\right)+3 \mu(A) U\right] .
\end{aligned}
$$


Case 2. If $E_{1} \subset A$ then, since $A$ does not contain both $E_{1}$ and $E_{2}$, there are elements in $z$ in $\overline{c o} f\left(E_{2} \backslash A\right)$ and $u$ in $U$ such that

$$
\begin{aligned}
x_{2} & =z \mu\left(E_{2}\right)+3 \mu\left(A \cap E_{2}\right) u \\
& =z \mu\left(E_{1} \cup E_{2}\right)-z \mu\left(E_{1}\right)+3 \mu\left(A \cap E_{2}\right) u .
\end{aligned}
$$

Also note that $x_{1} \in H_{f}\left(E_{1}\right) \subset \mu\left(E_{1}\right) U$. Thus $\left\|x_{1}-z \mu\left(E_{1}\right)\right\| \leqslant 2 \mu\left(E_{1}\right)$, and

$$
\begin{aligned}
x_{1}+x_{2} & \in \overline{\operatorname{co}} f\left(E_{2} \backslash A\right) \mu\left(E_{1} \cup E_{2}\right)+3 \mu\left(E_{1}\right) U+3 \mu\left(E_{2} \cap A\right) U \\
& \subset \overline{\operatorname{co}}\left[f\left(E_{1} \cup E_{2} \backslash A\right) \mu\left(E_{1} \cup E_{2}\right)+3 \mu(A) U\right] .
\end{aligned}
$$

The importance of the set function $H_{f}$ will now become apparent.

THEOREM 4.6. Let $(\Omega, \Sigma, \mu)$ be perfect. $A$ bounded weakly measurable function $f$ from $\Omega$ into the unit ball of $X$ is Pettis integrable if and only if $H_{f}(E) \neq \varnothing$ for every set $E$ of positive measure.

Proof. One direction of this is immediate: if $f$ is Pettis integrable then $\int_{E} f d \mu \in$ $H_{f}(E)$.

For the converse we apply Corollary 3.3. Let $B$ be any set of positive measure. If $\pi$ is a partition of $B$, then $\Sigma_{E \in \pi} H_{f}(E) \subset \Sigma_{E \in \pi} \overline{\operatorname{co}} f(E) \mu(E) \subset S(\pi, B)$. The superadditivity of $H_{f}$ shows that $\Sigma_{E \in \pi} H_{f}(E) \subset H_{f}(B)$. Hence $H_{f}(B) \cap S(\pi, B) \neq$ $\varnothing$ for all partitions $\pi$ of $B$, and $f$ is Pettis integrable.

Of course, since $G_{f}(E) \subset H_{f}(E)$, Theorems 4.4 and 4.6 are valid with $H_{f}(E)$ replaced by $G_{f}(E)$. We turn now to the set function $Q_{f}(E)$.

THEOREM 4.7. If $\mu(E)>0$, then

$$
G_{f}(E) \subset Q_{f}(E) \mu(E) \subset H_{f}(E) .
$$

Proof. First we show that $G_{f}(E) \subset Q_{f}(E) \mu(E)$. Let $x$ be in $G_{f}(E)$. For any functional $x^{*}$ in the unit ball of $X^{*}$ and for any positive number $\varepsilon<2$, let

$$
A=\left\{t \in E: x^{*} f(t) \leqslant x^{*}(x) / \mu(E)+\varepsilon\right\} .
$$

Observe that $x \in \overline{\operatorname{co}}[f(E \backslash A) \mu(E \backslash A)+\mu(A) U]$; hence

$$
\inf \left[x^{*} f(E \backslash A)\right] \mu(E \backslash A) \leqslant x^{*}(x)+\mu(A),
$$

and thus $\left[x^{*}(x) / \mu(E)+\varepsilon\right] \mu(E \backslash A) \leqslant x^{*}(x)+\mu(A)$. Solving this inequality for $\mu(A)$ gives

$$
\mu(A) \geqslant \frac{\varepsilon \mu(E)}{1+x^{*}(x) / \mu(E)+\varepsilon} .
$$

Observe that if $x^{*}(x) / \mu(E)+\varepsilon>1$ then $A=E$, and the conclusion $\mu(A)>$ $\varepsilon \mu(E) / 2$ holds trivially. On the other hand, if $x^{*}(x) / \mu(E)+\varepsilon<1$, then again $\mu(A)>\varepsilon \mu(E) / 2$. In either case

$$
\mu\left[t \in E: x^{*} f(t) \leqslant x^{*}(x) / \mu(E)+\varepsilon\right]>\varepsilon \mu(E) / 2,
$$

and $x / \mu(E) \in Q_{f}(E)$.

Now let $x$ be in $Q_{f}(E)$; it is apparent that $\left\|x^{*}\right\| \leqslant 1$. Let $A \subset E$. If $\mu(A)=\mu(E)$, then

$$
x \in U \subset \overline{\operatorname{co}}[f(E \backslash A)+(3 \mu(A) / \mu(E)) U] .
$$


If $\mu(A)<\mu(E)$, then for each $x^{*}$ in the unit ball of $X^{*}$ the inequality

$$
\mu\left[t \in E: x^{*} f(t) \leqslant x^{*}(x)+2 \mu(A) / \mu(E)\right]>\mu(A)
$$

holds. Hence there is a point $t$ in $E \backslash A$ such that $x^{*} f(t) \leqslant x^{*}(x)+2 \mu(A) / \mu(E)$, and thus

$$
x^{*}(x) \geqslant \inf \left[x^{*} f(E \backslash A)\right]-2 \mu(A) / \mu(E) .
$$

By the Hahn-Banach theorem, we have

$$
x \mu(E) \in \overline{\operatorname{co}}[f(E \backslash A) \mu(E)+2 \mu(A) U] \subset \overline{\operatorname{co}}[f(E \backslash A) \mu(E)+3 \mu(A) U] .
$$

Thus $x \mu(E) \in H_{f}(E)$, and this completes the proof.

5. Examples. In this section we present several examples of Pettis integrable functions and the set functions associated with them. The first example shows that even when $f$ is real-valued, the set $Q_{f}(E)$ need not be closed.

EXAMPLE 5.1. A function for which $Q_{f}(E)$ is not closed. Let $\mu$ represent Lebesgue measure on $\Omega=[0,1]$, and let $f: \Omega \rightarrow X$ be defined by $f(t)=\sqrt{t}$. Straightforward calculations show that

$$
G_{f}(\Omega)=[5 / 27,23 / 27], \quad Q_{f}(\Omega)=(1 / 8,1], \quad H_{f}(\Omega)=[1 / 12,1],
$$

and $\operatorname{cor}_{f}(\Omega)=[0,1]$. Notice that these sets are increasing and that each contains $\int_{\Omega} f d \mu=2 / 3$, as required by our work in the preceding section.

EXAMPLE 5.2. Let $\left(E_{n}\right)$ be a sequence of disjoint sets covering $\Omega=[0,1]$, such that $\mu\left(E_{n}\right)=2^{-n}$ for every integer $n$. Let $\left(e_{n}\right)$ be the unit vector basis of $l_{1}$ and define $f: \Omega \rightarrow l_{1}$ by

$$
f(t)=\sum_{n} \chi_{E_{n}}(t) e_{n}
$$

By Theorem 2.2, we know $\operatorname{cor}_{f}(\Omega)=\overline{\operatorname{co}}\left\{\int_{E_{n}} f d \mu / \mu\left(E_{n}\right)\right\}=\overline{\operatorname{co}}\left\{e_{n}\right\}$. In other words, $\operatorname{cor}_{f}(\Omega)$ consists of those elements of the unit sphere of $l_{1}$ with nonnegative entries. If $x=\left(x_{n}\right)$ is in $G_{f}(\Omega)$, then for each integer $n$ there must be nonnegative scalars $\left(\alpha_{i}\right)$ such that $\sum_{i \neq n} \alpha_{i}=1$ and $x \in\left(\sum_{i \neq n} \alpha_{i} e_{i}\right)\left(1-2^{-n}\right)+2^{-n} U$. Hence $x_{n} \leqslant 2^{-n}$ for each $n$. Since $G_{f}(\Omega) \subset \operatorname{cor}_{f}(\Omega)$, we see that the only element of $G_{f}(\Omega)$ is

$$
\sum_{n} 2^{-n} e_{n}=\int_{\Omega} d \mu
$$

In a similar manner, it is quite easily seen that $x=\left(x_{n}\right)$ is in $H_{f}(\Omega)$ if and only if $0 \leqslant x_{n} \leqslant 3 / 2^{n}$ and $\sum_{n} x_{n}=1$. Now consider $Q_{f}(\Omega)$. By letting $x^{*}=-c_{n}^{*} \in l_{\infty}$ and requiring

$$
u\left[t: x^{*} f(t) \leqslant x^{*}(x)+\varepsilon\right]>\varepsilon / 2,
$$

we find necessary conditions for $Q_{f}(\Omega)$ similar to those for $H_{f}(\Omega)$. If $x=\left(x_{n}\right)$ is in $Q_{f}(\Omega)$, then $0 \leqslant x_{n} \leqslant 2 / 2^{n}$ and $\Sigma_{n} x_{n}=1$. These conditions, however, are not sufficient; the element $e_{1}$ is not in $Q_{p}(\Omega)$, as we see by letting $x^{*}$ be the sequence $\langle-1,1,1,1, \ldots\rangle$ and letting $\varepsilon=3 / 2$.

EXAMPLE 5.3. Consider the function defined by $f(t)=\chi_{[0, t]} \in L_{\infty}$. To calculate the integral of $f$, let $\psi$ be in $L_{1}$, and let $x^{*}$ be the element of $L_{\infty}^{*}$ corresponding to $\psi$. 
For any number $b>0$, we have

$$
\begin{aligned}
\int_{0}^{b} x^{*} f(t) d \mu(t) & =\int_{0}^{b} \int_{0}^{t} \psi(s) d \mu(s) d \mu(t) \\
& =\int_{0}^{b} \int_{s}^{b} \psi(s) d \mu(t) d \mu(s)=\int_{0}^{b} \psi(s)(b-s) d \mu(s) .
\end{aligned}
$$

Hence $\int_{0}^{b} f(t) d \mu(t)=(b-s) \chi_{[0, b]}(s)$ and the integral is the element of $L_{\infty}$ with graph

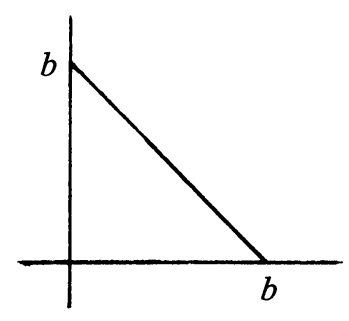

Note also that

$$
\frac{\int_{a}^{b} f(t) d \mu(t)}{b-a}=\chi_{[0, a)}(s)+\frac{b-s}{b-a} \chi_{[a, b]}(s),
$$

and the average value of $f$ over the interval $[a, b]$ is thus the element of $L_{\infty}$ with graph

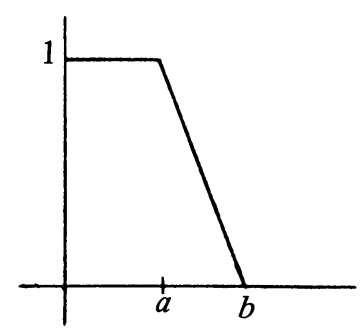

For each interval $[a, b]$, the average value of $f$ over $[a, b]$ is an element of $L_{\infty}$ with the properties

(1) $\phi(0)=1, \phi(1)=0$,

(2) $\phi$ is continuous,

(3) $\phi$ is decreasing.

Consequently, the average value of $f$ over any measurable set is an element of $L_{\infty}$ with the same properties. Theorem 2.2 guarantees that

$$
\operatorname{cor}_{f}(\Omega)=\overline{\operatorname{co}}\left\{\int_{E} f d \mu / \mu(E)\right\} ;
$$

hence every element of $\operatorname{cor}_{f}(\Omega)$ must satisfy (1)-(3). On the other hand, any function $\phi$ satisfying (1)-(3) can be uniformly approximated by piecewise-linear functions in $\operatorname{cor}_{f}(\Omega)$. The core of $f$ over $[0,1]$ therefore consists of all the elements of $L_{\infty}$ that satisfy conditions (1)-(3). 
Now let $\phi$ be in $H_{f}(\Omega)$; clearly $\phi$ must satisfy (1)-(3). For any interval $A$ in $[0,1]$ and for any number $\delta>0$ there is a convex sum $\sum \alpha_{i} f\left(t_{i}\right)$ such that $t_{i} \notin A$ and $\left\|\phi-\sum \alpha_{i} f\left(t_{i}\right)\right\|<\mu(A)+\delta$. The step function $\sum \alpha_{i} f\left(t_{i}\right)$ is constant on $A$. The variation of $\phi$ over $A$ is thus no greater than twice the length of $A$. It is easily seen that any element $\phi$ of $\operatorname{cor}_{f}(\Omega)$ that also satisfies condition

(4) $\operatorname{var}_{A} \phi \leqslant 2 \mu(A)$ for every interval $A$

is in $H_{f}(\Omega)$. Condition (4), of course, requires the functions in $H_{f}(\Omega)$ to be equicontinuous. The Arzela-Ascoli theorem now implies that $H_{f}(\Omega)$ is a compact subset of $L_{\infty}$, in agreement with Theorem 4.4.

6. A final question. We conclude this paper with a question: Is nonempty core sufficient to guarantee that a bounded weakly measurable function is Pettis integrable? An affirmative answer would be interesting in its own right and would have a number of interesting consequences. For example, this would show that Roman Pol's property (C) implies the Pettis Integral Property (PIP) for Banach spaces, settling a question raised by Edgar in [4]. While we feel that our work suggests an affirmative answer, at the moment the question is still open.

ADDED IN PROOF. The question above has recently been answered in the affirmative by M. Talagrand of the Equipe d'Analyse, Université Paris.

ACKNOWLEDGement. The author is grateful for the constant advice and support offered by Professor J. J. Uhl, Jr. during the preparation of the doctoral thesis from which this paper is taken.

\section{BIBLIOGRAPHY}

1. J. Diestel and J. J. Uhl, Jr., Vector measures, Math. Surveys, no. 15, Amer. Math. Soc., Providence, R. I., 1977.

2. N. Dunford and J. T. Schwartz, Linear operators. I, Interscience, New York, 1958.

3. G. A. Edgar, Measurability in a Banach space, Indiana Univ. Math. J. 26 (1977), 663-677.

4. __ Measurability in a Banach space. II, Indiana Univ. Math. J. (to appear).

5. D. H. Fremlin, Pointwise compact sets of measurable functions, Manuscripta Math. 15 (1975), 219-242.

6. D. M. Fremlin and M. Talagrand, A decomposition theorem for additive set-functions, with applications to Pettis integrals and ergodic means, Math. Z. 168 (1979), 117-142.

7. R. F. Geitz, Pettis integration, Proc. Amer. Math. Soc. (to appear).

8. R. C. James, Weak compactness and reflexivity, Israel J. Math. 2 (1964), 101-119.

9. B. J. Pettis, On integration in vector spaces, Trans. Amer. Math. Soc. 44 (1938), 277-304.

10. R. S. Phillips, Integration in a convex linear topological space, Trans. Amer. Math. Soc. 47 (1940), 114-145.

11. M. A. Rieffel, The Radon-Nikodym theorem for the Bochner integral, Trans. Amer. Math. Soc. 131 (1968), 466-487.

12. V. V. Sazonov, On perfect measures, Amer. Math. Soc. Transl. (2) 48 (1965), 229-254.

Department of Mathematics, Oberlin College, Oberlin, Ohto 44074 